\title{
Comprehensive preclinical evaluation of how cardiac safety profiles of potential COVID-19 drugs are modified by disease associated factors.
}

\author{
Clifford TeBay ${ }^{1}$, Jeffrey McArthur ${ }^{1}$, Melissa Mangala ${ }^{1}$, Nicholas Kerr ${ }^{1}$, STEWART \\ Heitmann ${ }^{1}$, Matthew Perry ${ }^{1}$, Monique Windley ${ }^{1}$, Jamie VaAndenberg ${ }^{1}$, and Adam Hill ${ }^{1}$ \\ ${ }^{1}$ Victor Chang Cardiac Research Institute
}

February 25, 2021

\begin{abstract}
Background and Purpose: Hydroxychloroquine and chloroquine, alone or in combination with azithromycin, have been proposed as therapies for COVID-19. However, there is currently scant and inconsistent data regarding their proarrhythmic potential in these patients. Moreover, their risk profile in the setting of altered physiological states encountered in patients with COVID-19 (i.e. febrile state, electrolyte imbalances, and/or acidosis) is unknown. Experimental approach: Potency of hERG block was measured using high-throughput electrophysiology in the presence of variable environmental factors. These potencies informed simulations to predict population risk profiles. Effects on cardiac repolarisation were verified in human induced pluripotent stem cell-derived cardiomyocytes (hiPSC-CM) from three separate individuals. Key Results: Chloroquine and hydroxychloroquine blocked hERG with IC50 of $1.47 \pm 0.07 \mu \mathrm{M}$ and $3.78 \pm 0.17 \mu \mathrm{M}$ respectively, indicating proarrhythmic risk at concentrations effective against SARS-CoV-2 in vitro and proposed in COVID-19 clinical trials. Hypokalaemia and hypermagnesemia increased potency of chloroquine and hydroxychloroquine, indicating increased proarrhythmic risk. Acidosis significantly reduced potency of all drugs (i.e. reduced proarrhythmic risk), whereas increased temperature decreased potency of chloroquine and hydroxychloroquine but increased potency for azithromycin. In silico simulations across genetically diverse populations predicted that $17 \%$ of individuals exhibit action potential durations $>500 \mathrm{~ms}$ at the highest proposed therapeutic levels, equating to significant QT prolongation. Conclusion and Implications: Significant proarrhythmic risk is predicted for hydroxychloroquine and chloroquine at doses proposed to treat COVID-19. Clinicians should carefully consider the risk of such treatments, and implement long term QT interval monitoring in trials, particularly in patients with electrolyte imbalances.
\end{abstract}

Comprehensive preclinical evaluation of how cardiac safety profiles of potential COVID-19 drugs are modified by disease associated factors.

Short title: Proarrhythmic risk of hydroxychloroquine, chloroquine and azithromycin.

Authors:

Clifford TeBay $\mathrm{BSc}^{1^{*}}$, Jeffrey R. McArthur $\mathrm{PhD}^{1,2^{*}}$, Melissa Mangala $\mathrm{PhD}^{1,3}$, Nicholas Kerr MBBS ${ }^{1,3}$, Stewart Heitmann $\mathrm{PhD}^{1}$, Matthew D. Perry $\mathrm{PhD}^{1,4}$, Monique J. Windley $\mathrm{PhD}^{1,3}$, Jamie I Vandenberg MBBS $\mathrm{PhD}^{1,3}$, Adam P. Hill $\mathrm{PhD}^{1,3}$

*These authors contributed equally to the work

Affiliations:

1. Victor Chang Cardiac Research Institute, Sydney, Australia.

2. Illawarra Health and Medical Research Institute, University of Wollongong, Australia. 
3. St. Vincent's Clinical school, UNSW Sydney, Sydney, Australia.

4. School of Medical Sciences, UNSW Sydney, Sydney, Australia.

\section{Corresponding author:}

Dr Adam P Hill

Victor Chang Cardiac Research Institute

405, Liverpool street

Darlinghurst

NSW 2020

Australia

Email: a.hill@victorchang.edu.au

Phone: +61 (0)2 92958686

Word count: 4042

Acknowledgements: We acknowledge support from the Victor Chang Cardiac Research Institute Innovation Centre, funded by the NSW Government.

Conflict of Interest statement: None

Keywords: Cardiac pharmacology, electrophysiology, safety pharmacology, COVID-19, Ion channels, Mathematical modelling

Data Availability Statement: The data that support the findings of this study are available from the corresponding author upon reasonable request. Some data may not be made available because of privacy or ethical restrictions.

\section{Summary:}

What is already known

Drugs that block hERG potassium channels in the heart have potential to cause cardiac arrhythmias

Hydroxychloroquine, chloroquine and azithromycin can block hERG potassium channels

What this study adds

Disease associated metabolic changes such as febrile temperature, acidosis and electrolyte imbalance modify hERG potency and hence proarrhythmic risk

Chronic exposure to hydroxychloroquine and azithromycin causes additional prolongation pf repolarisation compared to their acute effects

Clinical significance

There is significant proarrhythmic risk for hydroxychloroquine and chloroquine at doses being proposed to treat COVID-19

Clinicians should implement long term QT interval monitoring in trials, particularly in patients with electrolyte imbalances

Background and Purpose: 
Hydroxychloroquine and chloroquine, alone or in combination with azithromycin, have been proposed as therapies for COVID-19. However, there is currently scant and inconsistent data regarding their proarrhythmic potential in these patients. Moreover, their risk profile in the setting of altered physiological states encountered in patients with COVID-19 (i.e. febrile state, electrolyte imbalances, and/or acidosis) is unknown.

\section{Experimental approach:}

Potency of hERG block was measured using high-throughput electrophysiology in the presence of variable environmental factors. These potencies informed simulations to predict population risk profiles. Effects on cardiac repolarisation were verified in human induced pluripotent stem cell-derived cardiomyocytes (hiPSC$\mathrm{CM}$ ) from three separate individuals.

Key Results:

Chloroquine and hydroxychloroquine blocked hERG with $\mathrm{IC}_{50}$ of $1.47 \pm 0.07 \mu \mathrm{M}$ and $3.78 \pm 0.17 \mu \mathrm{M}$ respectively, indicating proarrhythmic risk at concentrations effective against SARS-CoV-2 in vitro and proposed in COVID-19 clinical trials. Hypokalaemia and hypermagnesemia increased potency of chloroquine and hydroxychloroquine, indicating increased proarrhythmic risk. Acidosis significantly reduced potency of all drugs (i.e. reduced proarrhythmic risk), whereas increased temperature decreased potency of chloroquine and hydroxychloroquine but increased potency for azithromycin. In silico simulations across genetically diverse populations predicted that $17 \%$ of individuals exhibit action potential durations $>500 \mathrm{~ms}$ at the highest proposed therapeutic levels, equating to significant QT prolongation.

\section{Conclusion and Implications:}

Significant proarrhythmic risk is predicted for hydroxychloroquine and chloroquine at doses proposed to treat COVID-19. Clinicians should carefully consider the risk of such treatments, and implement long term QT interval monitoring in trials, particularly in patients with electrolyte imbalances.

\section{Abbreviations:}

SARS-CoV-1, Severe Acute Respitatory Syndrome-Coronovirus-2; COVID-19, Coronavirus Disease 2019, aLQTS, acquired Long QT syndrome; hERG, human ether-a-go-go gene potassium channel; APD90, action potential duration at $90 \%$ repolarisation

\section{Introduction}

Severe acute respiratory syndrome coronavirus 2 (SARS-CoV-2) has to date infected 110 million people worldwide (December 2020), resulting in $\sim 2.5$ million deaths ( COVID-19 Dashboard, Johns Hopkins University). There are currently no small molecule medications approved to prevent or treat COVID-19. Throughout 2020, there was considerable interest in the use of hydroxychloroquine and chloroquine, two drugs used for treatment of lupus erythematosus, other inflammatory disorders and malaria, which are also effective in inhibiting replication of SARS-CoV-2 in vitro (1). Open-label observational studies purported to show therapeutic benefit for these drugs either when used as a monotherapy, or in combination with the macrolide antibiotic azithromycin (2). Consequently, the Food and Drug Administration (FDA) gave limited authorisation for emergency use of hydroxychloroquine in COVID-19 patients. However, this was subsequently been withdrawn with interim results from major clinical trials reporting negative results for efficacy against COVID-19 (3). Despite this, more than 150 clinical trials testing hydroxychloroquine and chloroquine, alone or in combination with azithromycin, for treatment of COVID-19 are still recruiting (www.clinicaltrials.gov), with many using doses several times higher than those recommended for their approved indications.

Significant concern exists around the cardiac safety profiles of hydroxychloroquine, chloroquine and azithromycin (4-6) as they are all known to prolong the QT interval on the electrocardiogram (ECG), primarily via block of the hERG/Kv11.1 potassium channel, and thus increase the risk of arrhythmias and sudden cardiac death (7). There are hundreds of cases of drug-induced arrhythmias, and cardiac arrest, associated with these drugs in the FDA's adverse events reporting system (FAERS; www.fda.gov). In the 
context of COVID-19 patients, this concern was most recently highlighted in a multinational registry analysis that showed increased risk of arrhythmias in patients treated with hydroxychloroquine and chloroquine, either with or without azithromycin (8). However, this study was subsequently retracted due to concerns over the veracity of the data (9). Consequently, there is scant and inconsistent data regarding the cardiac safety of these drugs at doses relevant to COVID-19.

In addition to the intrinsic risk associated with these drugs, many factors associated with the pathophysiology of COVID-19 may increase the risk of proarrhythmic activity. For example, fever, electrolyte changes including hypokalaemia and hypermagnesaemia, and systemic acidosis associated with respiratory failure, are common in COVID-19 patients $(8,10,11)$ and can all modify potency of hERG block and by extension proarrhythmic risk (12-15). Indeed, emerging evidence suggests that the QT prolongation observed for these drugs appears more profound in COVID-19 patients than in healthy individuals (5) suggesting a role for patient attributes in modifying proarrhythmic risk. Thus, there is a need for a thorough assessment of the cardiac safety profile of chloroquine, hydroxychloroquine and azithromycin in the context of changes in pathophysiological state associated with COVID-19, to guide clinical decision making and management of these patients.

Here, we have undertaken a multiplatform, preclinical cardiac safety evaluation combining high-throughput screening of hERG potency, in silico prediction of population risk, and analysis of repolarization changes in human induced pluripotent stem cell derived cardiomyocytes (hiPSC-CM) from multiple individuals to assess both acute and long term effects. Our data demonstrate a significant proarrhythmic risk for hydroxychloroquine and chloroquine at doses being proposed to treat COVID-19 and suggest that clinicians should implement long term QT interval monitoring in trials, particularly in patients with electrolyte imbalances (Graphical Abstract). Aside from specific relevance of our results to use of these drugs in treatment of COVID-19, this study also acts as a blueprint for how high-throughput in vitro screening, combined with in silico simulations, can be used to build reference datasets for how proarrhythmic risk of QT prolonging drugs can be modified by metabolic changes associated with disease in order to help guide clinicians in management of patients.

\section{Methods \\ Cell Culture:}

Chinese hamster ovary (CHO) cells stably expressing hERG (HERG-CHO) were purchased from American Type Culture Collection (ATCC reference PTA-6812, Manassas, VA). Cells were cultured in Hams F12 nutrient mix (ThermoFisher Scientific, Waltham, USA) supplemented with $5 \%$ fetal bovine serum (SigmaAldrich, Sydney, Australia) at $37^{\circ} \mathrm{C}$ with $5 \% \mathrm{CO}_{2}$. Cells were passaged using TrypLE ${ }^{\mathrm{TM}}$ Express Enzyme (ThermoFischer Scientific, Waltham, USA).

Human iPSC (hiPSC) lines, obtained from the Stanford Cardiovascular Institute Biobank, were maintained and differentiated as previously described (16). All lines were derived from donors between 15 and 50 years old. Lines $15 \mathrm{C} 1$ and $8 \mathrm{C} 1$ were from male subjects and line 142 from a female subject. HiPSC were maintained as colonies on Matrigel@ matrix (Corning) in mTeSR Plus cell culture medium (StemCell technologies) and passaged using ReLeSR (StemCell technologies). Differentiation to cardiomycoytes was performed using the STEMdiff Cardiomyocyte differentiation and maintenance Kit (StemCell Technologies). Between days 13-18, cardiomyocytes were dissociated by incubation in $0.2 \%$ Collagenase Type I (ThermoFisher) for 45 minutes at $37 \mathrm{degC}$ to break up the matrix, then incubated in $0.25 \%$ Trypsin with EDTA for 10 minutes at room temperature to break up cell clumps, before filtering through a $40 \mu \mathrm{m}$ cell strainer to obtain single hiPSC derived cardiomyocytes (hiPSC-CM).

\section{Patch-clamp electrophysiology}

Automated patch-clamp recordings were performed using a SyncroPatch 384PE (Nanion Technologies, Munich, Germany), with NPC-384 single hole medium resistance (4-5 M) chips as described previously (16). Prior to experiments, stably expressing hERG-CHO cells were dissociated with Accumax (Sigma-Aldrich, 
Sydney, Australia) and resuspended to a density of $\sim 180$ - 440K cells $/ \mathrm{mL}$ in chip fill solution, containing (in $\mathrm{mM}): 140 \mathrm{NaCl}, 5 \mathrm{KCl}, 10 \mathrm{HEPES}, 5$ Glucose, $\mathrm{pH} 7.4$ with $\mathrm{NaOH}(\sim 298 \mathrm{mOsm})$. Cells were incubated at $4^{\circ} \mathrm{C}$ for at least 1 hour prior to experiments. For whole cell recordings, the intracellular solution contained (in $\mathrm{mM}$ ): $110 \mathrm{KF}, 10 \mathrm{KCl}, 10 \mathrm{NaCl}, 10 \mathrm{HEPES}, 10 \mathrm{EGTA}, \mathrm{pH} 7.2\left({ }^{\sim} 285 \mathrm{mOsm}\right)$ and the standard extracellular solution contained (in $\mathrm{mM}$ ): $140 \mathrm{NaCl}, 5 \mathrm{KCl}, 2 \mathrm{CaCl}_{2}, 1 \mathrm{MgCl}_{2}, 10 \mathrm{HEPES}, 5$ Glucose, $\mathrm{pH} 7.4$ with $\mathrm{NaOH}(\sim 298 \mathrm{mOsm})$. To assess the impact of electrolyte changes on drug block, extracellular $\mathrm{KCl}$ or $\mathrm{MgCl}_{2}$ concentrations were varied from 3 to $7 \mathrm{mM}$ and 0.5 to $3 \mathrm{mM}$, respectively. For $\mathrm{pH}$ experiments, the $\mathrm{pH}$ was adjusted to $\mathrm{pH} 7.0$ or 6.5 . Cells were voltage clamped at a holding potential of $-80 \mathrm{mV}$ then depolarized to $40 \mathrm{mV}$ for $4 \mathrm{~s}$ followed by a $1 \mathrm{~s}$ test pulse to $-40 \mathrm{mV}$ to elicit hERG tail currents, with $15 \mathrm{~s}$ start-to-start interval. The degree of drug block of hERG current was assessed as the change in peak amplitude of the tail current after 12 minutes of drug application.

\section{Multi-electrode array recordings}

Extracellular field potentials were recorded from hiPSC-CM monolayers using the Maestro-APEX MEA system and AxIS software v2.5.1.10 software (Axion Biosystems, Atlanta, GA, USA) as previously described (16). Dissociated hiPSC-CM were spotted into Matrigel@ matrix coated wells of E-STIM+ Classic 48 well MEA plates (Axion Biosystems) at a density of 80,000 cells per well, using a robotic Maestro-APEX MEA system (Axion Biosystems, Atlanta, GA, USA). HiPSC-CM were initially maintained for 2 days in MEM alpha media (Gibco) supplemented with $10 \%$ foetal bovine serum (Sigma-Aldrich), before switching to RPMI 1640 medium (Thermo Fisher) containing B-27 supplement (Gibco, Thermo Fisher), supplemented to $1 \mathrm{mM}$ extracellular calcium concentration, for 20-30 days prior to recording. Extracellular field potentials were recorded from hiPSC-CM monolayers using the Maestro-APEX MEA system and AxIS software v2.5.1.10 software (Axion Biosystems, Atlanta, GA, USA). Monolayers, maintained at $37 \mathrm{deg} \mathrm{C}$ and $5 \% \mathrm{CO} 2$, were paced at $1 \mathrm{~Hz}$ and signals digitized at $12.5 \mathrm{kHz}$. Field potential durations were measured from the peak depolarization spike to the peak of the repolarization wave, using the CiPA analysis tool (Axion Biosystems, Atlanta, GA, USA). After 5 minutes of pacing equilibration, signals were recorded for 2-5 minutes under baseline conditions, after acute addition of drug ( $<2$ hours), and following 24 hours and 48 hours in the presence of hydroxychloroquine alone $(10 \mu \mathrm{M}$, nominal concentration) or in combination with azithromycin ( $5 \mu \mathrm{M}$, nominal concentration). Only wells showing properly paced signals at all time points were included in the analysis with field potential durations being measured from an average of 30 stable beast from a single electrode in each well. For MEA experiments examining the effect of kalaemic variation, cells were switched to Tyrode's solution 2 hours prior to recording (containing in mM: $140 \mathrm{NaCl}, 5.4$ or $3 \mathrm{KCl}, 1.8 \mathrm{CaCl}_{2}, 1$ $\mathrm{MgCl}_{2}, 10$ HEPES, 10 Glucose, adjusted with $\mathrm{NaOH}$ to $\mathrm{pH}$ 7.4) to allow for manipulation of $\left[\mathrm{K}^{+}\right]$.

\section{In silico modelling}

A population of $n=500$ human cardiac action potentials (AP) were simulated using the endocardial configuration of the ToR-Ord model (17) run in the Brain Dynamics Toolbox (18). To incorporate population variability in expression levels of the ion channels and electrogenic transporters that regulate cardiac excitability (i.e. the rhythmonome genes), the maximum conductance for each current (L-type calcium current, sodium current, transient outward current, late sodium current, rapid delayed rectifier (hERG) potassium current, slow delayed rectifier potassium current, inward rectifier potassium current, background potassium current, sodium calcium exchanger, sodium potassium pump, background sodium current, background calcium current, calcium pump, chloride current through calcium channel, background chloride current, calcium release from SR, calcium uptake to SR; Supplemental table 1) was multiplied by a conductance scalar $(G$ x) randomly drawn from a log-normal distribution $(19,20)$ with unit mean and variance of 0.05 , which we previously demonstrated can account for the range of QT intervals measured from normal and Long QT patient populations (21). Hypokalemia was simulated by manipulating parameterko in the model. The pharmacology of hydroxychloroquine were represented by an additional set of scaling factors, informed by the $\mathrm{IC}_{50}$ measured in our hERG screen, as well as data describing block of inward rectifier potassium current $\left(\mathrm{I}_{\mathrm{K} 1}\right)$, cardiac sodium current $\left(\mathrm{I}_{\mathrm{Na}}\right)$, and the cardiac L-type calcium current $\left(\mathrm{I}_{\mathrm{CaL}}\right)$ from the literature (22) (Supplemental table 2). Simulated myocytes were paced at $1 \mathrm{~Hz}$ and allowed to equilibrate for 300 beats 
prior to analysis. Source code: https://git.victorchang.edu.au/projects/CC/repos/.

\section{Data Analysis:}

Data analysis for the patch-clamp electrophysiology was performed using DataController384 V1.5.2 (Nanion Technologies) and Prism 8 (Graphpad Software, San Diego, CA, USA). Concentration response curves were fit with the Hill equation:

$$
\text { normalized } I_{\mathrm{hERG}} \text { inhibition }=\frac{[\mathrm{drug}]^{n}}{\mathrm{IC}_{50}{ }^{n}+[\text { drug }]^{n}}
$$

where [drug] is the drug concentration, $n$ is the Hill coefficient, and $I C_{50}$ is the half-maximal inhibitory concentration.

\section{Statistical Methods:}

For comparison of $\mathrm{IC}_{50} \mathrm{~s}$, a Brown-Forsythe and Welch ANOVA with a post-hoc Games-Howell multiple comparison was performed with significance reported as * for $\mathrm{p}[?] \quad 0.05,{ }^{* *}$ for $\mathrm{p}$ [?] $0.005,{ }^{* * *}$ for $\mathrm{p}$ [?] 0.0005 , and $* * * *$ for $\mathrm{p}[?] 0.0001$.

In MEA experiments, data were averaged from $n$ individual MEA wells for each hiPSC line, with individual line data also shown. A repeated measures two-way ANOVA test was performed to determine statistical significance between drug treatments and over time, with significance reported as P [?] 0.05.

\section{Results}

Chloroquine, hydroxychloroquine and azithromycin block hERG at physiological temperatures

The potency of each compound for hERG block was determined using a Syncropatch PE384 automated patch clamp system. Typical current traces and timecourse of block for ${ }^{\sim} \mathrm{IC}_{50}$ doses of chloroquine (1 $\mu \mathrm{M})$, hydroxychloroquine $(3 \mu \mathrm{M})$ and azithromycin $(100 \mu \mathrm{M})$ are shown in Figures 1A-C. The potency of hERG inhibition $\left(\mathrm{IC}_{50} \pm \mathrm{SEM}\right.$ ) was $1.47 \pm 0.07 \mu \mathrm{M}, 3.78 \pm 0.18 \mu \mathrm{M}$, and $280.1 \pm 14.7 \mu \mathrm{M}$ for chloroquine, hydroxychloroquine and azithromycin respectively (Figure 1D).

\section{Influence of electrolyte imbalance, acidosis and temperature on block of hERG.}

For each drug, we measured how the potency of hERG block was modified by i) kalaemic variation (3/5/7 $\mathrm{mM} \mathrm{K}^{+}$representing hypo-, normo-, and hyper-kalaemia, respectively); ii) variation in extracellular magnesium (0.5/1/2 $\mathrm{mM} \mathrm{Mg}^{2+}$ representing hypo-, normo-, and hyper-magnesemia, respectively); iii) $\mathrm{pH}$ ( $\mathrm{pH} 7.4$, 7 and 6.5 representing normal, acidosis and severe acidosis, respectively); and iv) temperature $\left(37^{\circ} \mathrm{C}\right.$ and $42{ }^{\circ} \mathrm{C}$ representing physiological and febrile temperature). Hypokalaemia significantly increased potency of hERG block for both chloroquine and hydroxychloroquine (1.4- and 2.0-fold increase, respectively) while hyperkalaemia significantly decreased potency of chloroquine (2.1-fold decrease). Azithromycin was insensitive to kalaemic variation (Figure 2, Table 1). Hypomagnesemia had no significant effect on potency of any of the drugs. However, hypermagnesemia significantly increased potency of hERG block for both chloroquine and hydroxychloroquine (2.8-fold and 1.3-fold respectively (Figure 2, Table 1)). Reduction in $\mathrm{pH}$ from 7.4 to 7 significantly decreased potency of all drugs (3.4-, 5.0- and 2.2-fold for chloroquine, hydroxychloroquine, and azithromycin respectively) and this trend continued with further reductions in potency observed at $\mathrm{pH}$ 6.5 (Figure 2, Table 1). Finally, increased temperature $\left(42{ }^{\circ} \mathrm{C}\right)$ significantly decreased potency of chloroquine and hydroxychloroquine by 1.9 - and 1.6-fold respectively, but increased potency of azithromycin by 3.9 -fold (Figure 2, Table 1).

Effect of hydroxychloroquine and azithromycin on cardiac repolarization in iPSC cardiomyocytes

Data from our screen of hERG potency showed only a subtle (hydroxychloroquine), or non-significant (chloroquine), additive effect of azithromycin (Supplementary figure 2). In order to measure the effects of the 
combination of drugs directly on cardiac repolarisation, as well as to assess whether there are any potentially proarrhythmic effects that manifest with longer term exposure, we measured field potential duration, a surrogate of the QT interval, in hiPSC derived cardiomyocytes from three normal patients. Representative field potentials for hydroxychloroquine alone (Figure 3A) and in combination with azithromycin (Figure 3B) are shown for (i) baseline; (ii), acutely after drug addition; (iii) $24 \mathrm{hrs}$ and (iv) $48 \mathrm{hrs}$ after drug addition. Summary data for all three lines over time is presented in Figure 3C (individual technical replicates for each cell line are presented in Supplementary Figure 4). There was no significant difference in the extent of prolongation of repolarisation between hydroxychloroquine alone, or in combination with azithromycin (Repeated measures two-way ANOVA; $\mathrm{p}=0.109$ ). However, there was a significant time dependent effect (Repeated measures two-way ANOVA; $\mathrm{p}=0.027$ ), indicating an accumulation of block with longer term exposure to the drugs. Additionally, hydroxychloroquine and the combination of hydroxychloroquine and azithromycin caused cessation of beating or loss of capture in multiple wells (Table 2). This is consistent with previous reports showing that profound drug-induced disruption of repolarisation, corresponding to 75 $\%$ or more block of IKr, can result in cessation of beating or occurrence of arrhythmia (23). Next, we assessed the effect of kalaemic variation on prolongation of repolarisation by hydroxychloroquine. $10 \mu \mathrm{M}$ hydroxychloroquine caused significantly more prolongation in hypokalaemia $\left(3 \mathrm{mM} \mathrm{K} \mathrm{K}^{+}, 7.7 \pm 1.1 \%\right.$ prolongation $(\mathrm{SEM} ; \mathrm{n}=16)$ ) than normokalaemia $\left(5.4 \mathrm{mM} \mathrm{K}^{+}, 3.3 \pm 0.5 \%\right.$ prolongation (SEM; $\left.\mathrm{n}=17\right)$ ) demonstrating that the hypokalaemia-dependent increase in potency of hydroxychloroquine against hERG, as measured in our Syncropatch screens (Figure 2), manifests in a more severe prolongation of cardiac repolarisation.

\section{Population level predictions of QT prolonging effects for hydroxychloroquine and hypokalaemia}

Our hiPSC-CM data suggests differences in drug effects between individual patient lines as well as a more pronounced prolongation of repolarisation in the context of hypokalamia. To interrogate this further, we performed population level simulations of the cardiac action potential to assess the variability in drug response between individual subjects and to illustrate the consequences of the electrolyte-dependent changes in hERG potency. In addition to the kalaemic-dependence of potency of hydroxychloroquine against hERG measured in our assays, these simulations also incorporate block of the inward rectifier potassium current $\left(\mathrm{I}_{\mathrm{K} 1}\right)$, the cardiac sodium current $\left(\mathrm{I}_{\mathrm{Na}}\right)$, and the cardiac L-type calcium current $\left(\mathrm{I}_{\mathrm{CaL}}\right)$ from the literature (22). However, it should be noted that the contribution of this multichannel pharmacology to the overall effect on repolarisation is relatively small, since the degree of block at the highest dose of hydroxychloroquine simulated $(3000 \mathrm{ng} / \mathrm{ml})$ were $4.1 \%, 20 \%$ and $7.3 \%$ for $\mathrm{I}_{\mathrm{CaL}}, \mathrm{I}_{\mathrm{K} 1}$ and $\mathrm{I}_{\mathrm{Na}}$ respectively, compared to $70 \%$ block of hERG at the same concentration (see Supplementary table 2). Populations of action potentials representing baseline (no drug, normal electrolytes), $1000 \mathrm{ng} / \mathrm{ml}$ hydroxychloroquine and hydroxychloroquine + mild hypokalaemia are shown in Figure 4A. Frequency distribution histograms of repolarisation duration illustrating the effect of hypokalaemia on population response to $1000 \mathrm{ng} / \mathrm{ml}$ and $3000 \mathrm{ng} / \mathrm{ml}$ hydroxychloroquine (an estimate of mean plasma $\mathrm{C}_{\max }$ and maximum plasma $\mathrm{C}_{\max }$ respectively, see Discussion) are shown in Figure 4B-D and summarised in Figure 4E. In normokalaemia, the time to $90 \%$ repolarisation $\left(\mathrm{APD}_{90}\right)$ was increased to $355 \pm 49 \mathrm{~ms}($ mean $\pm \mathrm{SD}$ ) and $4444 \pm 60 \mathrm{~ms}$ for $1000 \mathrm{ng} / \mathrm{ml}$ and $3000 \mathrm{ng} / \mathrm{ml}$ hydroxychloroquine respectively compared to baseline $(274 \pm 40 \mathrm{~ms})$. Furthermore, within these distributions, a range in the severity of prolongation of repolarisation was observed, even for those individuals with the same baseline $\mathrm{APD}_{90} \mathrm{~s}$ (Supplementary Movie 1). Mild hypokalaemia further increased the duration of repolarisation to $383 \pm 53 \mathrm{~ms}$ and $481 \pm 63 \mathrm{~ms}$ for $1000 \mathrm{ng} / \mathrm{ml}$ and $3000 \mathrm{ng} / \mathrm{ml}$ hydroxychloroquine. The percentage of the population with $\mathrm{APD}_{90}>500 \mathrm{~ms}$ in response to $3000 \mathrm{ng} / \mathrm{ml}$ hydroxychloroquine increased from $17.4 \%$ in normokalaemia to $34.8 \%$ in hypokalaemia, reflecting a significant increase in proarrhythmic risk associated with mild hypokalaemia.

\section{Discussion}

This study demonstrates that both chloroquine and hydroxychloroquine carry cardiac safety risks that are of significant concern in the context of therapeutic doses being proposed for treatment of COVID-19. Furthermore, the proarrhythmic risk associated with block of hERG channels by these compounds can be exacerbated in patients by electrolyte abnormalities such as hypokalaemia and hypermagnesemia. Co-administration of 
azithromycin with hydroxychloroquine did not further increase prolongation of repolarisation to hydroxychloroquine alone in the short term. However, there was a time-dependent increase in prolongation observed with long term exposure, supporting the need for long term QT interval monitoring in these patients.

\section{Hydroxychloroquine and chloroquine block hERG at doses relevant to COVID-19.}

Recent in vitro studies demonstrated $\mathrm{EC}_{50}$ s for inhibition of viral replication at $24 / 48$ hours of $0.72 \mu \mathrm{M} / 6.14$ $\mu \mathrm{M}$ for hydroxychloroquine (1) and suggested that these concentrations could be achieved with a dosing regimen of 2 X $400 \mathrm{mg}$ loading/2 X $200 \mathrm{mg}$ daily. However, other trials have proposed significantly higher dosing. For example, the Norwegian arm of the WHO's SOLIDARITY trial had a dosing regimen of 2 X $800 \mathrm{mg}$ loading and then 2 X $400 \mathrm{mg}$ daily (24). Based on Phase I trials in patients with melanoma this would be expected to result in an average peak blood $\mathrm{C}_{\max }$ of $2000-3000 \mathrm{ng} / \mathrm{ml}$, with some patients reaching 4000-6000 ng/ml (25). Assuming $50 \%$ serum protein binding (26), this would correspond to an average peak plasma $\mathrm{C}_{\max }$ of $1000-1500 \mathrm{ng} / \mathrm{ml}(3-4.5 \mu \mathrm{M})$ but up to $4000-6000 \mathrm{ng} / \mathrm{ml}(6-9 \mu \mathrm{M})$ in extreme cases. In comparison, our analysis showed $\mathrm{IC}_{50}$ values for $\mathrm{hERG}$ block of $3.7 \mu \mathrm{M}$ for hydroxychloroquine at physiological temperature (Figure 1).

For chloroquine, Yao et al. measured an $\mathrm{EC}_{50}$, for inhibition of viral replication at 24/48 hours, of 5.47 $\mu \mathrm{M} / 23.9 \mu \mathrm{M}$ and suggested that these concentrations could be reached with a daily $500 \mathrm{mg}$ dose (1). Various $\mathrm{PK} / \mathrm{PD}$ studies broadly support this with $450 \mathrm{mg}$ daily dose for three days yielding a median $\mathrm{C}_{\max }$ of 376 $\mu \mathrm{g} / \mathrm{L}(1.2 \mu \mathrm{M})$ in one study (27) and long term $500 \mathrm{mg}$ daily dose shown to equilibrate to $>10 \mu \mathrm{M}$ plasma concentration (equal to $\sim 3000 \mu \mathrm{g} / \mathrm{L}$ ) in a second study (28). However, initial guidelines issued for use of chloroquine in COVID-19 patients recommended twice this dose (29) and this is reflected in proposed trials (30). For comparison, we measured $\mathrm{IC}_{50}$ for hERG block of $1.5 \mu \mathrm{M}$ for chloroquine (Figure 1 ). Together, these data suggest significant hERG block, and hence proarrhythmic risk, at doses relevant to COVID19. For example, a plasma $\mathrm{C}_{\max }$ of $\sim 3 \mu \mathrm{M}$ (a realistic estimate for either drug based on the above), would cause 43 and $67 \%$ block of hERG for hydroxychloroquine and chloroquine. This use case therefore falls well short of the $30-45$ fold safety margins $\left(\mathrm{C}_{\max }\right.$ :hERG $\left.\mathrm{IC}_{50}\right)$ that have been proposed as a safe threshold in preclinical assays $(31,32)$. Furthermore, our analysis does not take into account that both chloroquine and hydroxychloroquine have been reported to accumulate in the heart (33), meaning cardiac hERG channels could be exposed to much higher concentrations. Our data therefore support the conclusion that chloroquine and hydroxychloroquine carry cardiac safety liabilities that are of significant concern at the doses being proposed for treatment of COVID-19.

\section{Metabolic changes associated with COVID-19 alter proarrhythmic risk associated with hERG block}

The potency of compounds that block the hERG potassium channel and hence infer proarrhythmic risk is known to be sensitive to a variety of pathophysiological states including temperature, electrolyte concentrations and $\mathrm{pH}$ (12-15). This is an important consideration in COVID-19 patients. For example, febrile temperature is reported in $>90 \%$ of hospitalised COVID-19 cases $(8,34)$, hypokalaemia in up to $41 \%$ of patients (10) and acidosis in $9 \%$ of cases and $30 \%$ of non-survivors (8). Furthermore, acute kidney injury has been reported in $15 \%$ of in-patient cases and $50 \%$ of non-survivors (8) with potential for further electrolyte imbalances including hyperkalaemia and hypermagnesemia. There is evidence that COVID-19 patients treated with hydroxychloroquine/chloroquine show more severe QTc prolongation than healthy individuals (5), and those patients with acute renal failure exhibited the most extreme effects (35). It is therefore critical that we understand how these altered physiological states in patients affect hERG potency and hence potential proarrhythmic propensity of these drugs.

While it is accepted that each of these factors can affect potency of hERG block, reports of how they do so are conflicting (e.g. acidic $\mathrm{pH}$ has been reported to both increase and decrease potency of hERG block $(15,36))$ and can differ between drugs (e.g. the effect of temperature on block of hERG is drug-specific (12)). It was therefore important to examine how environmental factors in COVID-19 specifically modify the proarrhythmic risk profile of hydroxychloroquine and chloroquine. Our results show that the effects of 
temperature were drug specific, decreasing potency for hydroxychloroquine and chloroquine but increasing potency for azithromycin. However, even with elevated temperature, the $\mathrm{IC}_{50}$ for azithromycin $(\sim 70 \mu \mathrm{M})$ was an order of magnitude higher than the likely plasma $\mathrm{C}_{\max }(37)$. These results suggest that febrile temperatures should not raise additional concern specific to drug-induced arrhythmia. Similarly, lowering $\mathrm{pH}$ to $\mathrm{pH} \mathrm{7,} \mathrm{consistent} \mathrm{with} \mathrm{severe} \mathrm{systemic} \mathrm{acidosis} \mathrm{that} \mathrm{might} \mathrm{be} \mathrm{observed} \mathrm{with} \mathrm{respiratory} \mathrm{or} \mathrm{multi-organ}$ failure, significantly reduced the potency of all drugs between 3 and 5-fold, again suggesting that additional concern relating to drug-induced arrhythmias may be mitigated by profound acidosis. An exception to this might be if local acidosis in the myocardium, in the context of coronary occlusion for example (38), led to spatial differences in the degree of hERG block and hence regional gradients of repolarisation that could act as an electrical substrate for reentry and arrhythmogenesis (39).

Conversely, our data show that some electrolyte imbalances, specifically hypermagnesemia and hypokalemia could increase proarrhythmic risk. Hypermagnesemia significantly increased potency of hERG block (3-fold and 1.4-fold for chloroquine and hydroxychloroquine respectively). Likewise, hypokalaemia increased potency of both drugs (1.4-fold and 2-fold for chloroquine and hydroxychloroquine respectively), and this increase in hERG potency translated to more pronounced prolongation of repolarisation of iPSC cardiomyocytes in the context of reduced extracellular $\mathrm{K}^{+}$(Figure 3D). These data suggest that particular attention should be given to monitoring drug-induced QT changes COVID-19 patients with these electrolyte abnormalities. Also of potential clinical relevance, our finding that hypermagnesemia increases potency of hERG block suggests that if intravenous magnesium is given as a therapy to avoid or to treat torsade de pointes in COVID-19 patients, then great care should be taken not to overshoot the normal physiologic range.

\section{Prediction of population risk profile}

hERG potency screens, such as that described above, are a mandated requirement during preclinical safety screening (40). However these screens only give an overall indication of proarrhythmic risk (41). The severity of any given individual's response to QT prolonging drugs is dependent on their specific genetic background since variability in expression of rhythmonome genes between individuals can alter their 'repolarisation reserve' capacity, making them more or less susceptible to drug-induced arrhythmias (42). To predict the extent and variability of response to the hERG block we have measured for hydroxychloroquine, we integrated our ion channel screen data into population level simulations of action potentials from individuals with different genetic backgrounds. This approach allows us to both derive a quantitative indication of population risk, as well as assess how the changes in potency that occur as a result of electrolyte imbalance shift the population risk. Our data show that concentrations of hydroxychloroquine relevant to COVID-19 are predicted to cause significant prolongation of repolarisation. Moreover, the range of responses within the population is broad, with some individuals displaying repolarisation times in the normal range, even in response to the highest doses of hydroxychloroquine, while at the other tail of the population, some individuals are predicted to be at high risk, with repolarisation times $>500 \mathrm{~ms}$ (a threshold chosen since QTc $>500 \mathrm{~ms}$ is associated with significantly increased risk of life-threatening cardiac events (43). Of potential significant to management of these patients, a normal $\mathrm{APD}_{90}$ was not necessarily predictive of the severity of prolongation of repolarisation observed in the presence of drug. Rather, there are many combinations of genetic background that give rise to the same baseline phenotype, that respond differently to hERG block, depending on their repolarisation reserve. This is consistent with the observation that some patients showing the most pronounced QT prolongation after treatment with hydroxychloroquine had normal QT at baseline (5) emphasising the need for longer term QTc monitoring. The simulations also demonstrate how even mild hypokalaemia can shift the population risk profile. For example, a reduction in potassium of $0.5 \mathrm{mM}$ (from $5 \mathrm{mM}$ to $4.5 \mathrm{mM}$ ) increased the mean $\mathrm{APD}_{90}$ of the population in the context of $3000 \mathrm{ng} / \mathrm{ml}$ hydroxychloroquine by an additional 40 $\mathrm{ms}$ and increased the number of individuals with repolarisation times in the high risk range $(>500 \mathrm{~ms})$ from $17 \%$ to $35 \%$. Overall, our population simulations largely reflect recent data from cohort studies that report similar variability and extent of response (5), thus supporting the utility of the model for population risk prediction, particularly in the context of COVID-19 associated risk factors.

Prolongation of repolarisation accumulates with long term exposure. 
A treatment options that is currently being evaluated is the combination of hydroxychloroquine with azithromycin (44). Studies in small groups of patients reported significant QTc prolongation in those treated with this combination, with $11 \%$ of patients showing severe prolongation of $>500 \mathrm{~ms}(5)$ - though with no incidence of arrhythmia. Another retrospective cohort study of 1438 patients demonstrated that cardiac arrest was significantly more likely in COVID-19 patients receiving the combination of drugs, but not in patients receiving either drug alone (6). However, the authors pointed out that there were potential limitations with this observation. In the absence of randomized clinical trial data, there therefore exists some degree of uncertainty about whether there is an additive QT effect of the combination therapy.

In our study, we first assessed whether the potency of either hydroxychloroquine or chloroquine against hERG was altered when administered with $5 \mu \mathrm{M}$ azithromycin, corresponding to the plasma $\mathrm{C}_{\max }$ of an IV dosing regime of $500 \mathrm{mg}$ per day (37). Azithromycin caused only subtle (hydroxychloroquine) or insignificant changes in the extent of hERG block when compared to each drug in isolation (Supplementary figure 2), consistent with a purely additive effect of the drug. This approach however does not take into account the possibility that azithromycin can i) accumulate in cells and tissue to much higher concentrations than in plasma (45); and ii) may also act on other ion channel involved in cardiac repolarisation in addition to hERG. To address this, we measured the time dependent effect of hydroxychloroquine, with and without azithromycin, on prolongation of repolarisation over 48 hours in hiPSC derived cardiomyocytes from three separate individuals. Our data showed that while there was no statistically significant difference observed between the effects of the two drug treatments, there was a significant increase in prolongation observed over time. This is consistent with previously published pharmacokinetic studies reporting accumulation of both hydroxychloroquine $(33,46)$ and azithromycin $(45)$ in tissue. Azithromycin has also been shown to potentiate the late sodium current with longer term exposure (47), which would also prolong repolarisation and exacerbate the proarrhythmic risk associated with hERG block. The time dependent effects observed in our experiments therefore likely result from a combination of each of these effects. We also observed clear variability in the magnitude of response to the drugs between iPSC lines derived from different patients. This is consistent with previously published data from our group (48) and others (49) where we have measured the transcript levels of cardiac rhythmonome genes and show that the degree of prolongation measured on multielectrode arrays is dependent on the genetic background of the individual, specifically the level of expression of the KCNH2 gene, coding for the hERG $\mathrm{K}^{+}$channel. Overall, these data support that longer term QTc monitoring should be implemented in COVID-19 patients administered hydroxychloroquine or chloroquine, either alone or in combination with azithromycin, in addition to baseline ECG screening recommended in recent guidelines (4).

\section{Limitations}

In the absence of detailed pharmacokinetic and pharmacodynamic studies in COVID-19 patients, plasma concentrations corresponding to the doses of hydroxychloroquine and chloroquine that are currently undergoing evaluation in clinical trials were best estimates based on existing literature in other patient groups. Similarly, the concentration of azithromycin used in our study, while corresponding to the reported plasma $\mathrm{C}_{\max }$, does not take into account the possibility of tissue accumulation in patients, potentially underestimating the degree of hERG block and hence proarrhythmic risk. Since the initial completion of this work, other chemical entities have shown antiviral activity against SARS-CoV2 in vitro, including 90 positive hits from the Prestwick chemical library (50). This study is therefore not an exhaustive analysis of small molecules that could be trialled for treatment of COVID-19. However, it represents, to our knowledge, the most comprehensive in vitro dataset for how hERG-related proarrhythmia can be modified by disease associated factors and as such provides a new blueprint for high throughput in vitro analysis of disease associated proarrhythmic risk.

\section{Conclusions}

There have been more than 200 clinical trials registered for hydroxychloroquine and/or chloroquine each considering different dosing regimes and different combinations with drugs including azithromycin (www.clinicaltrials.gov). Although these drugs are generally well tolerated when used for their approved 
indications, there is ample evidence that both can cause QT prolongation and carry a risk of drug-induced arrhythmia (7). Preliminary data from observational studies points to QT prolonging effects being more pronounced in COVID-19 patients than heathy individuals (5). Furthermore, patient comorbidities such as renal failure may add to the proarrhythmic risk in COVID-19 patients (35). In the current absence of gold-standard randomized clinical trial data that is sufficiently powered to assess cardiac safety, coupled with the urgent need to identify potential therapies for COVID-19, there is a critical need for a comprehensive preclinical assessment of the cardiac safety profile of these drugs, in relation to COVID-19 patients. Our data indicate a significant proarrhythmic risk for hydroxychloroquine and chloroquine at doses being proposed to treat COVID-19 that can be increased by electrolyte imbalances reported in these patients (Graphical Abstract). Thus, it would be prudent to implement long term QT interval monitoring in these patients, particularly those with electrolyte imbalances. More broadly, our study demonstrates how the proarrhythmic risk associated with drugs that prolong cardiac repolarisation can be modified by disease related metabolic changes that occur in patients and acts as a blueprint for how high-throughput in vitro screening, combined with in silico simulations can integrate these effects into preclinical screening for proarrhythmic risk.

\section{References}

1. Yao X, Ye F, Zhang M, et al. In Vitro Antiviral Activity and Projection of Optimized Dosing Design of Hydroxychloroquine for the Treatment of Severe Acute Respiratory Syndrome Coronavirus 2 (SARS-CoV-2). Clin. Infect. Dis. 2020.

2. Gautret P, Lagier J-C, Parola P, et al. Hydroxychloroquine and azithromycin as a treatment of COVID-19: results of an open-label non-randomized clinical trial. Int. J. Antimicrob. Agents 2020:105949.

3. WHO Solidarity Trial Consortium, Pan H, Peto R, et al. Repurposed Antiviral Drugs for Covid-19 Interim WHO Solidarity Trial Results. N Engl J Med 2020.

4. Giudicessi JR, Noseworthy PA, Friedman PA, Ackerman MJ. Urgent Guidance for Navigating and Circumventing the QTc-Prolonging and Torsadogenic Potential of Possible Pharmacotherapies for Coronavirus Disease 19 (COVID-19). Mayo Clin. Proc. 2020;95:1213-1221.

5. Chorin E, Dai M, Shulman E, et al. The QT interval in patients with COVID-19 treated with hydroxychloroquine and azithromycin. Nat Med 2020;26:808-809.

6. Rosenberg ES, Dufort EM, Udo T, et al. Association of Treatment With Hydroxychloroquine or Azithromycin With In-Hospital Mortality in Patients With COVID-19 in New York State. JAMA 2020.

7. Woosley RL, Heise CW, Romero KA. www.Crediblemeds.org.

8. Zhou F, Yu T, Du R, et al. Clinical course and risk factors for mortality of adult inpatients with COVID-19 in Wuhan, China: a retrospective cohort study. Lancet 2020;395:1054-1062.

9. Mehra MR, Ruschitzka F, Patel AN. Retraction-Hydroxychloroquine or chloroquine with or without a macrolide for treatment of COVID-19: a multinational registry analysis. The Lancet 2020:1-1.

10. Lippi G, South AM, Henry BM. Electrolyte imbalances in patients with severe coronavirus disease 2019 (COVID-19). Ann Clin Biochem 2020;57:262-265.

11. Alfano G, Ferrari A, Fontana F, et al. Hypokalemia in Patients with COVID-19. medRXiv 2020:1-18.

12. Windley MJ, Lee W, Vandenberg JI, Hill AP. The Temperature Dependence of Kinetics Associated with Drug Block of hERG Channels Is Compound-Specific and an Important Factor for Proarrhythmic Risk Prediction. Mol Pharmacol 2018;94:760-769.

13. Barrows B, Cheung K, Bialobrzeski T, Foster J, Schulze J, Miller A. Extracellular potassium dependency of block of HERG by quinidine and cisapride is primarily determined by the permeant ion and not by inactivation. Channels (Austin) 2009;3:239-248. 
14. Po SS, Wang DW, Yang IC, Johnson JP, Nie L, Bennett PB. Modulation of HERG potassium channels by extracellular magnesium and quinidine. J. Cardiovasc. Pharmacol. 1999;33:181-185.

15. Du CY, Harchi El A, Zhang YH, Orchard CH, Hancox JC. Pharmacological inhibition of the hERG potassium channel is modulated by extracellular but not intracellular acidosis. J Cardiovasc Electrophysiol 2011;22:1163-1170.

16. Perry MD, Ng CA, Mangala MM, et al. Pharmacological activation of IKr in models of long QT Type 2 risks overcorrection of repolarization. Cardiovasc. Res. 2019;81:299.

17. Tomek J, Bueno-Orovio A, Passini E, et al. Development, calibration, and validation of a novel human ventricular myocyte model in health, disease, and drug block. Elife 2019;8.

18. Heitmann S, Aburn MJ, Neurocomputing MB, 2018. The Brain Dynamics Toolbox for Matlab. Neurocomputing 2018;315:82-88.

19. Sadrieh A, Domanski L, Pitt-Francis J, et al. Multiscale cardiac modelling reveals the origins of notched $\mathrm{T}$ waves in long QT syndrome type 2. Nat Commun 2014;5:5069.

20. Sadrieh A, Mann SA, Subbiah RN, et al. Quantifying the origins of population variability in cardiac electrical activity through sensitivity analysis of the electrocardiogram. J Physiol (Lond) 2013;591:42074222 .

21. Mann SA, Imtiaz M, Winbo A, et al. Convergence of models of human ventricular myocyte electrophysiology after global optimization to recapitulate clinical long QT phenotypes. J Mol Cell Cardiol $2016 ; 100: 25-34$.

22. Wang G, Lu C-J, Trafford AW, et al. Mechanistic insights into ventricular arrhythmogenesis of hydroxychloroquine and azithromycin for the treatment of COVID-19. bioRxiv 2020;3:e208857-31.

23. Blinova K, Stohlman J, Vicente J, et al. Comprehensive Translational Assessment of Human Induced Pluripotent Stem Cell Derived Cardiomyocytes for Evaluating Drug-Induced Arrhythmias. Toxicol. Sci. 2016;155:kfw200-247.

24. Shukla AM, Archibald LK, Wagle Shukla A, Mehta HJ, Cherabuddi K. Chloroquine and hydroxychloroquine in the context of COVID-19. DIC 2020;9.

25. Rangwala R, Leone R, Chang YC, et al. Phase I trial of hydroxychloroquine with dose-intense temozolomide in patients with advanced solid tumors and melanoma. Autophagy 2014;10:1369-1379.

26. McLachlan AJ, Cutler DJ, Tett SE. Plasma protein binding of the enantiomers of hydroxychloroquine and metabolites. Eur. J. Clin. Pharmacol. 1993;44:481-484.

27. Karunajeewa HA, Salman S, Mueller I, et al. Pharmacokinetics of chloroquine and monodesethylchloroquine in pregnancy. AAC 2010;54:1186-1192.

28. Mackenzie AH. Dose refinements in long-term therapy of rheumatoid arthritis with antimalarials. The American Journal of Medicine 1983;75:40-45.

29. Gendrot M, Javelle E, Clerc A, Savini H, Pradines B. Chloroquine as a prophylactic agent against COVID-19? Int. J. Antimicrob. Agents 2020;55:105980.

30. Fihn SD, Perencevich E, Bradley SM. Caution Needed on the Use of Chloroquine and Hydroxychloroquine for Coronavirus Disease 2019. JAMA Netw Open 2020;3:e209035-14.

31. Redfern WS, Carlsson L, Davis AS, et al. Relationships between preclinical cardiac electrophysiology, clinical QT interval prolongation and torsade de pointes for a broad range of drugs: Evidence for a provisional safety margin in drug development. Cardiovasc. Res. 2003;58:32. 
32. Gintant G. An evaluation of hERG current assay performance: Translating preclinical safety studies to clinical QT prolongation. Pharmacology \& Therapeutics 2011;129:109-119.

33. McChesney EW, Banks WF, Fabian RJ. Tissue distribution of chloroquine, hydroxychloroquine, and desethylchloroquine in the rat. Toxicol. Appl. Pharmacol. 1967;10:501-513.

34. Pan L, Mu M, Yang P, et al. Clinical Characteristics of COVID-19 Patients With Digestive Symptoms in Hubei, China: A Descriptive, Cross-Sectional, Multicenter Study. Am. J. Gastroenterol. 2020;115:766-773.

35. Chorin E, Dai M, Shulman E, et al. The QT Interval in Patients with SARS-CoV-2 Infection Treated with Hydroxychloroquine/Azithromycin. medRXiv 2020:2020.04.02.20047050.

36. Wang Y, Guo J, Perissinotti LL, et al. Role of the $\mathrm{pH}$ in state-dependent blockade of hERG currents. Sci Rep 2016;6:32536.

37. Pfizer. Zithromax IV. 2013:1-16.

38. Fleet WF, Johnson TA, Graebner CA, Gettes LS. Effect of serial brief ischemic episodes on extracellular $\mathrm{K}+, \mathrm{pH}$, and activation in the pig. Circulation 1985;72:922-932.

39. Clayton RH, Holden AV. Dispersion of cardiac action potential duration and the initiation of re-entry: a computational study. Biomed Eng Online 2005;4:11.

40. Food and Drug Administration, HHS. International Conference on Harmonisation; guidance on S7B Nonclinical Evaluation of the Potential for Delayed Ventricular Repolarization (QT Interval Prolongation) by Human Pharmaceuticals; availability. Notice. Fed Regist 2005;70:61133-61134.

41. Yap YG, Camm AJ. Drug induced QT prolongation and torsades de pointes. Heart 2003;89:1363-1372.

42. Behr ER, Roden D. Drug-induced arrhythmia: pharmacogenomic prescribing? Eur Heart J 2013;34:8995.

43. Sauer AJ, Moss AJ, McNitt S, et al. Long QT syndrome in adults. J Am Coll Cardiol 2007;49:329-337.

44. Gautret P, Lagier J-C, Parola P, et al. Hydroxychloroquine and azithromycin as a treatment of COVID19: results of an open-label non-randomized clinical trial. Int. J. Antimicrob. Agents 2020:105949.

45. Araujo FG, Shepard RM, Remington JS. In vivo activity of the macrolide antibiotics azithromycin, roxithromycin and spiramycin against Toxoplasma gondii. Eur. J. Clin. Microbiol. Infect. Dis. 1991;10:519524.

46. Tett SE, Cutler DJ, Day RO, Brown KF. A dose-ranging study of the pharmacokinetics of hydroxychloroquine following intravenous administration to healthy volunteers. Br J Clin Pharmacol 1988;26:303313.

47. Yang Z, Prinsen JK, Bersell KR, et al. Azithromycin Causes a Novel Proarrhythmic Syndrome. Circ Arrhythm Electrophysiol 2017;10.

48. Ballouz S, Mangala MM, Perry MD, et al. Co-expression of calcium and hERG potassium channels reduces the incidence of proarrhythmic events. Cardiovasc. Res. 2020.

49. Stillitano F, Hansen J, Kong CW, Elife IK, 2017. Modeling susceptibility to drug-induced long QT with a panel of subject-specific induced pluripotent stem cells. eLife 2017;6:e19406.

50. Touret F, Gilles M, Barral K, et al. In vitro screening of a FDA approved chemical library reveals potential inhibitors of SARS-CoV-2 replication. Sci Rep 2020;10:13093.

Table 1

\begin{tabular}{llll}
\hline Drug + Condition & I“50 $(\mu \mathrm{M})$ & SEM & Significance \\
\hline Chloroquine (control) & 1.473 & 0.066 &
\end{tabular}




\begin{tabular}{|c|c|c|c|}
\hline Drug + Condition & $\mathrm{I}^{\text {"N5}} 50(\mu \mathrm{M})$ & SEM & Significance \\
\hline $3 \mathrm{mM}$ Potassium & 1.080 & 0.061 & $* * *$ \\
\hline 7 mM Potassium & 3.091 & 0.252 & $* * * *$ \\
\hline $0.5 \mathrm{mM}$ Magnesium & 1.475 & 0.151 & ns \\
\hline $3 \mathrm{mM}$ Magnesium & 0.530 & 0.052 & $* * * *$ \\
\hline pH 7.0 & 5.017 & 0.306 & $* * * *$ \\
\hline $\mathrm{pH} 6.5$ & 62.335 & 3.390 & $* * * *$ \\
\hline $42^{\circ} \mathrm{C}$ & 2.786 & 0.237 & $* * * *$ \\
\hline Coadministration & 1.440 & 0.065 & $\mathrm{~ns}$ \\
\hline Hydroxychloroquine (control) & 3.781 & 0.184 & \\
\hline $3 \mathrm{mM}$ Potassium & 1.846 & 0.109 & $* * * *$ \\
\hline $7 \mathrm{mM}$ Potassium & 4.140 & 0.286 & ns \\
\hline $0.5 \mathrm{mM}$ Magnesium & 3.818 & 0.212 & ns \\
\hline 3 mM Magnesium & 2.874 & 0.173 & $*$ \\
\hline $\mathrm{pH} 7.0$ & 18.758 & 1.250 & $* * * *$ \\
\hline $\mathrm{pH} 6.5$ & 127.548 & 10.493 & $* * * *$ \\
\hline $42{ }^{\circ} \mathrm{C}$ & 5.894 & 0.426 & $* * *$ \\
\hline Coadministration & 2.807 & 0.080 & $* * * *$ \\
\hline Azithromycin (control) & 280.078 & 14.650 & \\
\hline $3 \mathrm{mM}$ Potassium & 232.923 & 20.559 & ns \\
\hline 7 mM Potassium & 254.692 & 17.801 & ns \\
\hline $0.5 \mathrm{mM}$ Magnesium & 266.608 & 18.307 & ns \\
\hline 3 mM Magnesium & 220.088 & 18.083 & ns \\
\hline pH 7.0 & 610.490 & 57.489 & $* * * *$ \\
\hline $\mathrm{pH} 6.5$ & 1157.749 & 112.820 & $* * * *$ \\
\hline $42{ }^{\circ} \mathrm{C}$ & 72.264 & 7.746 & $* * * *$ \\
\hline
\end{tabular}

Significance: * - p [?] 0.05, *** - p [?] 0.0005, **** - P [?]0.0001

Table 1: Statistical comparisons of IC50s for chloroquine, hydroxychloroquine and azithromycin in the presence of COVID-19 related factors.

Table 2

\begin{tabular}{llllll}
\hline & Hydroxychloroquine & Hydroxychloroquine & Hydroxychloroquine & Hydroxychloroquine + Azithromycin & Hydr \\
\hline Line ID & Acute & 24 hours & 48 hours & Acute & 24 ho \\
$8 \mathrm{C} 1$ & $0 / 7$ & $1 / 7$ & $1 / 7$ & $0 / 6$ & $0 / 6$ \\
$15 \mathrm{C} 1$ & $1 / 4$ & $2 / 4$ & $0 / 4$ & $1 / 6$ & $2 / 6$ \\
$142 \mathrm{C} 1$ & $0 / 6$ & $1 / 6$ & $0 / 6$ & $0 / 6$ & $1 / 6$ \\
\hline
\end{tabular}

Table 2: Frequency of observed cessation of beating/loss of capture on MEA in response to either $10 \mu \mathrm{M}$ hydroxychloroquine alone, or in combination with $5 \mu \mathrm{M}$ azithromycin. 
Ai.

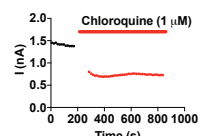

Time (s)

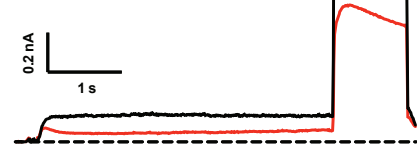

ii.

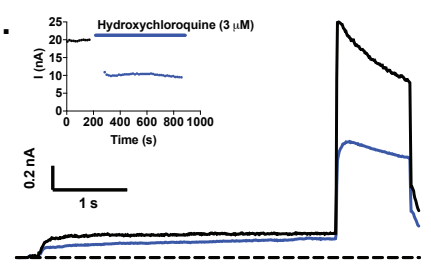

iii.

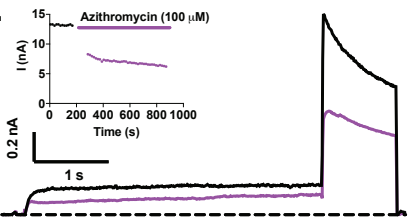

B.

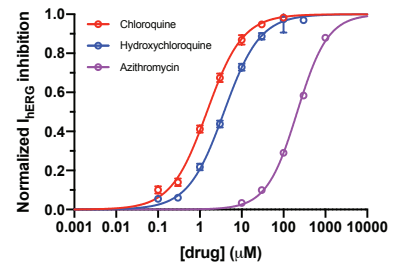

Figure 1 - Potency of hERG block by chloroquine, hydroxychloroquine and azithromycin. A) Typical example of hERG currents showing block by an approximate IC dose of i) chloroquine, ii) hydroxychloroquine, and iii) azithromycin. Timecourse of block is displayed inset. Cells were voltage clamped at a holding potential of $-80 \mathrm{mV}$ then depolarized to $40 \mathrm{mV}$ for $4 \mathrm{~s}$ followed by a $1 \mathrm{~s}$ test pulse to $-40 \mathrm{mV}$ to elicit tail currents, with $15 \mathrm{~s}$ start-to-start interval. B) Dose response curves for inhibition of hERG currents.IC s0 $_{50} \pm$ SEM for chloroquine (red), hydroxychloroquine (blue), and azithromycin (purple) were $1.47 \pm 0.07 \mu \mathrm{M}, 3.8 \pm 0.2 \mu \mathrm{M}$, and $212.4 \pm 10 \mu \mathrm{M}$ respectively. 
A.

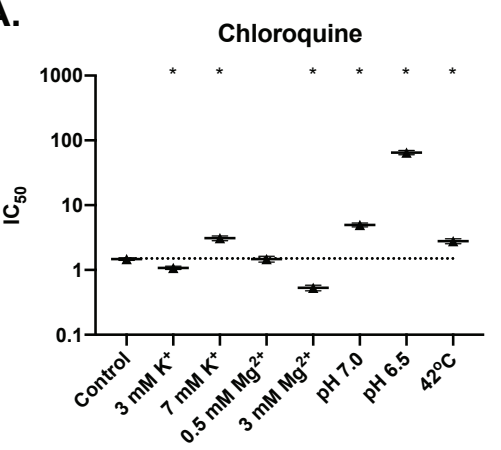

B.

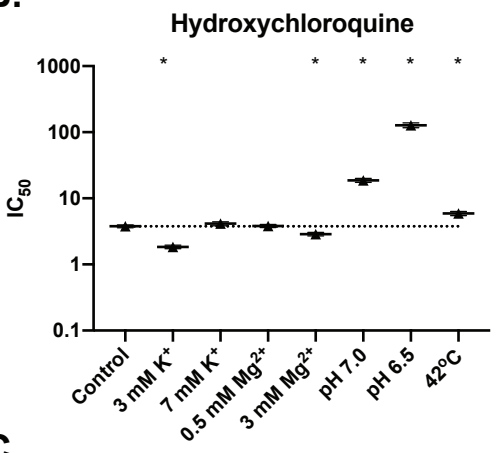

C.

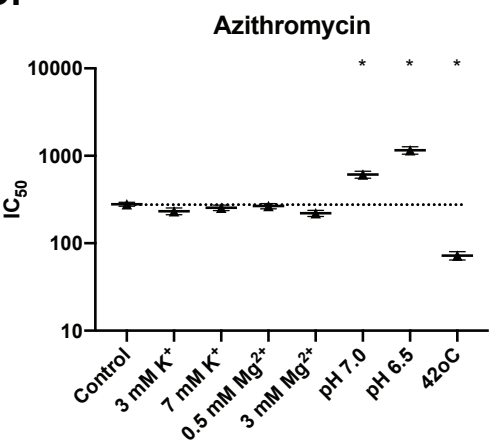

Figure 2 - Effect of electrolyte concentration, acidosis and temperature on potency of hERG block by chloroquine, hydroxychloroquine and azithromycin. Changes in $\mathrm{IC}_{50}$ derived from full dose response curves (Supplementary figure 1) as a function of COVID-19 related changes in physiological state for chloroquine (A), hydroxychloroquine (B) and azithromycin (C). Significant changes are indicated with asterisk. Full details of descriptive data and statistical analysis are presented in Table 1. 

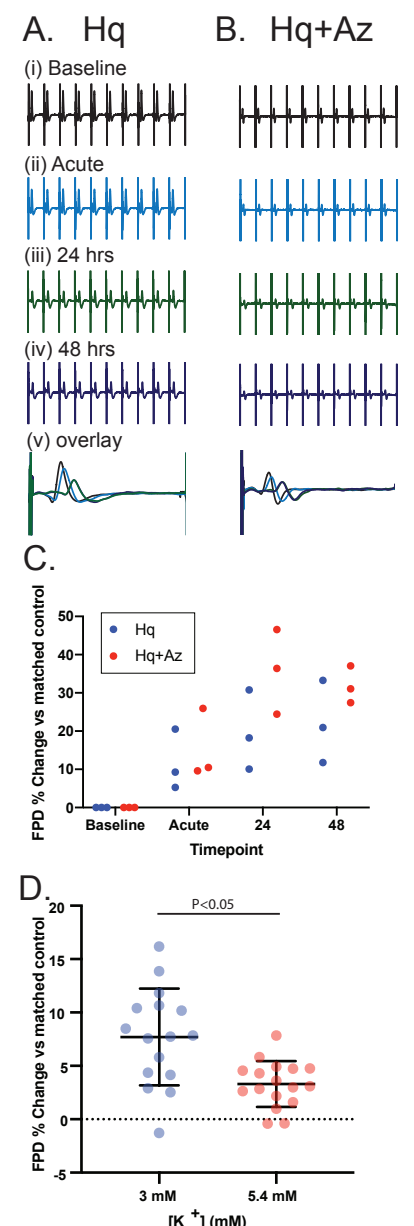

Figure 3 - Effect of hydroxychloroquine and azithromycin on cardiac repolarisation in iPSC cardiomyocytes. Typical examples of field potentials paced at $60 \mathrm{bpm}$ for iPSC cardiomyocytes exposed to hydroxychloroquine alone (A) or in combination with azithromycin (B). Data for baseline (i), acute application (ii), 24 hours (iii), and 48 hours (iv) are shown, in addition to an overlay of all timepoints (v). C) Summary data showing FPD

prolongation for three separate iPSC lines where each datapoint is an average of the technical replicates for that line. D) Effect of kalaemic variation on prolongation of repolarisation by hydroxychloroquine.

\section{Hosted file}

Figure4_new_2.pdf available at https://authorea.com/users/398216/articles/510865comprehensive-preclinical-evaluation-of-how-cardiac-safety-profiles-of-potential-covid19-drugs-are-modified-by-disease-associated-factors 